Cahiers de recherches médiévales

Journal of medieval studies

$8 \mid 2001$

La protection spirituelle au Moyen Âge

\title{
Le nom protecteur
}

Patrice Beck

\section{OpenEdition}

Journals

Édition électronique

URL : https://journals.openedition.org/crm/395

DOI : $10.4000 / \mathrm{crm} .395$

ISSN : 1955-2424

Éditeur

Honoré Champion

Édition imprimée

Date de publication : 15 janvier 2001

Pagination : 165-174

ISSN : 1272-9752

\section{Référence électronique}

Patrice Beck, « Le nom protecteur », Cahiers de recherches médiévales [En ligne], 8| 2001, mis en ligne le 13 mars 2008, consulté le 15 décembre 2022. URL : http://journals.openedition.org/crm/395 ; DOI https://doi.org/10.4000/crm.395

Ce document a été généré automatiquement le 15 décembre 2022.

Tous droits réservés 


\section{Le nom protecteur}

\section{Patrice Beck}

1 Pour les historiens comme pour les anthropologues, les sociologues et les psychologues, la dénomination est un acte universel, fondateur de chaque individu et de toute société.

2 Les analystes expliquent qu'elle est une nécessité pour structurer mentalement l'homme, car «l'usage du nom propre... instaure entre les personnes une discontinuité succédant à la confusion régnante entre les individus biologiques réduits à leurs attributs naturels $»^{1}$. C'est ce que constatent les anthropologues des sociétés africaines traditionnelles où il est rare « que l'on donne un nom à l'enfant dès sa naissance [...] car [...] le doute plane sur le caractère humain du bébé comme sur son désir de rester icibas " ${ }^{2}$. De même, " prénommer est un acte central du baptême, aussi bien du rituel de la conversion des adultes que de l'admission des nouveaux-nés dans la communauté chrétienne $»^{3}$. Le baptême est " une seconde naissance ou, mieux encore, la véritable naissance $»^{4}$, car « Dieu connaît chacun par son nom [...] et, en [...] appelant chacun par son nom, le Dieu de Jésus-Christ [...] le fait exister $»^{5}$. Ce rite de passage, sous la forme culturelle propre à l'église d'Occident, est bien présent dans les récits du Moyen-Âge, tant profanes qu'ecclésiastiques, dans les romans comme dans les exempla : ainsi «Lion et Bras, que l'ermite avait [...] instruits dans l'amour et la sainte foi de Jésus-Christ sans pour autant les avoir baptisés car il n'avait pas ce qui lui était nécessaire, étaient vêtus de feuilles d'arbre et de peaux de bête ${ }^{6} »$; ainsi encore cet autre enfant qui, «lorsqu'il eut reçu un nom, [...] exulta de joie et dès lors marcha dressé sur ses pieds alors qu'auparavant il s'était traîné sur le sol $»^{7}$. Il est avéré que sans le baptême et la dation du nom qui en est un point essentiel, l'être ne fait pas partie de la société des hommes, est condamné à une existence bestiale; après la mort, sans le nom de baptême qui s'inscrit aux cieux dans le Grand Livre de vie et qui sert à l'identifier au temps de la résurrection, le défunt hante les vivants 8 .

Il est non moins évident que la dénomination sert autant à classer et situer socialement qu'à individualiser, qu'elle est un vocabulaire de la parenté dont elle préserve la cohérence et protège l'intégrité, dont elle augmente l'efficacité en prolongeant la parenté de sang par la parenté spirituelle. Le nom en effet s'hérite comme le patrimoine et tout manquement apparait choquant, selon des principes dont la sagesse 
et l'ancienneté sont au moins bibliques, comme le rapporte l'Évangile selon Saint Luc: « et il advint, le huitième jour, qu'ils vinrent pour circoncire l'enfant. On voulait l'appeler Zacharie, du nom de son père mais, prenant la parole, sa mère dit : «non, il s'appellera Jean ». Et on lui dit : «il n'y a personne de ta parenté qui porte ce nom ». Et l'on demandait par signes au père comment il voulait qu'on l'appelât. Celui-ci demanda une tablette et écrivit : «Jean est son nom »; et ils en furent tous étonnés »9.

4 L'exigence est également germanique. Le procédé le plus répandu ${ }^{10}$ joue sur la bipartition des noms et consiste en une répétition d'un des deux éléments : ainsi les deux frères Lüdeger et Lüdegast, ou le Sigfrid fils de Sigemund et de Sigelinde de la "Chanson des Nibelungen ${ }^{11}$. Cette histoire de Burgondes a été mise par écrit au $\mathrm{XIII}^{\mathrm{e}}$ siècle et ce mode de désignation n'est plus alors qu'un procédé littéraire car, dans les pratiques, que des études sérielles récentes ont révélées au niveau européen ${ }^{12}$, il est surtout actif au haut Moyen-Âge, perd de sa lisibilité avant l'an mil. Il est remplacé par un nouveau système onomastique qui devient majoritaire à la fin du XIII ${ }^{e}$ siècle et s'appuie sur l'usage à la fois d'un patronyme et d'un stock familial de noms, déformables sans doute sous formes hypocoristiques mais insécables, transmis du père au fils ou du grand-père paternel au petit-fils pour les aînés, des oncles aux neveux pour les puînés ${ }^{13}$. Cette nouvelle forme de désignation est un repère social reconnu et utilisé dans les intrigues romancées : « N'ayez longuement compagnon en chemin ou en logis que vous ne demandiez son nom car par le nom on connaît l'homme ", écrit Chrétien de Troyes dans son Perceval ${ }^{14} \mathrm{et}$, à la fin du XIV siècle, le héros du Roman de Mélusine oppose son nom aux chevaliers menaçants : « vous le saurez donc et vous ferez demi-tour, malgré que vous en avez, je suis Geoffroy de Lusignan $»^{15}$.

5 Les ruptures dans la transmission posent problème et les accidents, ceux que les mortalités provoquent par exemple, doivent être réparés : c'est ainsi qu'en Limousin au XII ${ }^{\mathrm{e}}$ siècle, dans la Chronique de Geoffroy de Vigeois, un Ademarus, vicomte de Limoges, épouse une Turenne et prénomme un de ses fils Boso car il risquait de devoir recueillir la succession de son oncle maternel Boso, vicomte de Turenne; mais cette éventualité ne s'étant pas produite et Boso étant resté en revanche le seul fils vivant d'Adémarus, on changea son nom de Boso en celui d'Ademarus car il était destiné à recueillir alors la succession de son propre père ${ }^{16}$. C'est aussi le cas dans la Toscane $d u \mathrm{XV}^{\mathrm{e}}$ siècle où plus du quart des enfants de la bourgeoisie florentine étaient débaptisés afin que le nom survive malgré les vicissitudes démographiques et que soit donc évitée une rupture de la mémoire lignagère ${ }^{17}$.

6 Enfin, si les règles d'appellation des sociétés traditionnelles situent et intègrent l'individu dans la communauté sociale et dans sa parenté, si elles établissent une relation forte entre les vivants et les morts, donc entre le visible et l'invisible, le matériel et le sacré, elles procèdent inévitablement de l'action magique, transmettent des vertus, installent des protections, que ce soit le nom des héros, « des saints ou des ancêtres qui assurent la fonction de protecteurs des hommes vivants et morts $»^{18}$. «Le nom n'est-il pas l'équivalent d'un don magique [...] et [...] protéger l'enfant en lui donnant le nom adéquat n'est-il pas la fonction première de la nomination ? $»^{19}$.

Quelle soit première, seconde ou troisième, cette fonction est souvent sollicitée, dans les chroniques comme dans les romans, pour expliquer des choix de dénomination. C'est tantôt les seules vertus du sang ou les qualités du héros qui sont mobilisées, comme pour ces enfants des Nibelungen auxquels, à l'un on « donna le nom de Gunther qui était celui de son oncle [et dont] il n'avait point lieu de rougir [car] heureux cet 
enfant s'il devait marcher sur les traces de ses parents Burgondes » et à l'autre, «le nom de Sigfrid le héros, [...] pour l'amour de ce dernier $»^{20}$. C'est aussi le cas de ce Jean Tristan nommé ainsi vers 1450 dans le Livre de Baudoin comte de Flandre «à cause des douleurs qu'il avait causé à sa mère : - par Dieu! dit la mère, ce nom me plaît! Qu'il égale le mérite du vaillant Tristan... $»^{21}$. Et c'est ce qui pousse Ardaubourg, roi de Jérusalem, à prendre au baptême le nom d'Amaury son vainqueur : une manière de lui faire allégeance, de se placer sous sa protection et de s'approprier un peu de sa force, car « il dit aux seigneurs que ce n'était que justice qu'il portât son nom puisque c'était lui qui l'avait vaincu $»^{22}$.

8 Mais les références profanes sont surtout intégrées aux modèles religieux de vertus: ainsi l'enfant à naître de La belle Hélène de Constantinople devait-il porter «le nom de Titus, en l'honneur de Titus qui vengea à la pointe de l'épée la mort de notre Sauveur Jésus-Christ ou, si c'était une fille, celui d'Hélène, en l'honneur de cette sainte femme, Hélène, à qui apparut la Sainte Croix élevée pour notre rédemption ${ }^{23}$.

Mieux encore, la dédicace, la consécration même, de l'enfant à Dieu par l'entremise de l'un de ses saints, assure le transfert des compétences, guide le nommé dans la vie, le prédestine aux actions d'éclat. Ainsi dans le Livre de Baudoin, "la noble reine sentit venir les douleurs de l'accouchement, qui durèrent quatre jours et trois nuits. En implorant l'aide de Dieu et de Saint Jean-Baptiste, elle accoucha d'un beau fils que l'on nomma Jean car auparavant elle avait dit que si ce devait être un fils, on le nommerait ainsi, que si c'était une fille elle serait nommée Jeanne, et qu'elle consacrerait l'enfant qu'elle aurait à Saint Jean-Baptiste. En sortant du sein de sa mère, cet enfant nommé Jean, dont Dieu permit la naissance, portait une croix vermeille sur l'épaule droite, ce qui signifiait qu'il était venu au monde par la grâce de Dieu et qu'un jour il serait roi » ${ }^{24}$.

Autour de la dénomination, se pressent donc beaucoup de personnes et se mêlent bien des enjeux. Comment, en attribuant le nom, contenter tout le monde, protéger et rassurer efficacement l'ego et l'âme, la famille et la société, les vivants et les morts, le profane et le spirituel?

\section{La dénomination des personnes: entre intérêts profanes et exigences spirituelles}

11 Entre nécessités individuelles et collectives, la contradiction n'est qu'apparente car les déterminants sont de toute façon sociaux. «Comme le choix du conjoint, l'attribution du prénom à un enfant est en principe aléatoire puisque laissée au libre choix des parents. Mais cette liberté officielle recouvre une multitude de normes, de règles implicites qui orientent le choix et répondent à d'autres préoccupations que celle de désigner quelqu'un dans sa singularité. Même ceux qui croient avoir décidé selon leur humeur et leur goût, subissent les contraintes de la mode et les convenances de leur milieu. Le paradoxe de la tyrannie de l'originalité est qu'elle renforce le conformisme social. On croit faire un choix à contre-courant alors qu'on répercute avec un parfait mimétisme les normes esthétiques de son milieu social et de son époque $»^{25}$. Quant au surnom, il est rarement et pas longtemps personnel, il est surtout et dès l'origine nom de famille et de lignage, procède largement d'un choix collectivement accepté et même déterminé, les référents les plus usités étant le nom du père (nomen paternum), le lieu de 
provenance ou d'enracinement (anthropo-toponymes), le titre porté ou la fonction exercée ${ }^{26}$.

L'opposition principale n'est pas non plus tant entre les intérêts privés de la famille et ceux, publics, des princes. Pour les administrateurs des personnes et des biens, un seul souci : celui de bien prélever l'impôt et donc d'identifier, au mieux certes mais moins des individus que des feux, des unités de résidence et de production et donc de prélèvement fiscal. Comme l'ont montré les recherches d'A. Lefèvre-Teillard ${ }^{27}$ sur les actes normatifs, longtemps la loi fut muette en la matière : l'ordonnance qu'entend instituer en 1456 Charles VII, afin de poursuivre comme faussaire ceux qui changent de nom sans autorisation, est seulement prévue pour la Normandie et n'est pas même promulguée face à la vive réaction du Parlement. Quand l'État s'agite vraiment, c'est fort tardivement, au XVI ${ }^{\text {e }}$ siècle, et c'est d'abord pour confirmer le rôle que l'Église tient depuis longtemps d'enregistrer les actes de baptême (donc de naissance), de mariage et de décès : l'édit de Villers-Cotterêts de 1539, dans son article 51, ne porte en effet obligation que de tenir les registres paroissiaux. C'est la Révolution qui définira en la matière le droit, un droit minimal, confirmant à la sphère privée le privilège de nommer.

De fait, l'opposition fondamentale réside entre les exigences de la parenté, par essence segmentaires et privées, et les aspirations dogmatiques de l'Église chrétienne poussant à l'unicité, à l'universalité.

Le problème s'est immédiatement posé: Saint Jean Chrysostome, évêque de Constantinople de 397 à 407, félicite les chrétiens qui donnent à leurs enfants non pas le nom de leurs aïeux mais celui des saints et il blâme ceux qui se soustraient à cet usage pratiqué, dit-il, par les premiers fidèles ${ }^{28}$. Il est toujours d'actualité au XVIII ${ }^{\mathrm{e}}$ siècle: Girard de Villethierry, un prêtre, écrit en 1781 dans son traité de La vie des gens mariés ou les obligations de ceux qui s'engagent dans le mariage, prouvées par l'Ecriture, par les Saints Pères et par les Conciles ${ }^{29}$ que, dans le choix des noms à donner aux enfants, tous soient "guidés par des sentiments de piété et de religion; qu'ils aient dessin, en leur choisissant un Saint pour patron, de les engager à imiter ses vertus, et à le suivre dans les voies du salut... Ce sont là les véritables motifs qui doivent déterminer les Fidèles à donner les noms aux enfants qu'ils tiennent sur les Fonts du baptême. Tous les autres qu'ils peuvent se proposer, n'étant ordinairement fondés que sur des raisons de famille et sur des intérêts temporels, ne sont pas légitimes et ne doivent point être considérés dans le christianisme».

Un conflit donc, mais pas la guerre car le pouvoir religieux a su en la matière, comme le pouvoir civil, se faire longtemps discret: l'Église, n'a fait que suggérer, conseiller, éventuellement orienter les choix mais elle n'a jamais légiféré, dans le souci de ne pas heurter violemment les coutumes ancestrales car le choix du nom est avant tout et de tout temps familial ${ }^{30}$.

\section{Nom de naissance, nom de baptême}

De fait, si l'institution des noms de baptême succède immédiatement à l'usage d'imposer le nom dans la cérémonie de la circoncision, le huitième jour après la naissance ${ }^{31}$, le baptême reste longtemps différé: pendant le haut Moyen-Âge, il concerne des adultes qui se convertissent et se donne lors des fêtes solennelles de Pâques et de la Pentecôte. Aussi, la dénomination est d'abord un rite d'agrégation à la 
famille et le choix reste parental même après que le pédobaptisme se soit généralisé avec les réformes carolingienne puis grégorienne des $\mathrm{IX}^{\mathrm{e}}$ puis $\mathrm{XI}^{\mathrm{e}}-\mathrm{XII}{ }^{\mathrm{e}}$ siècles. Dans les chansons de gestes du XII ${ }^{e}$ siècle comme dans les romans des $\mathrm{XIV}^{\mathrm{e}}$ et $\mathrm{XV}^{\mathrm{e}}$ siècles, la substitution du nom soulignant la conversion, effet littéraire si commode pour évoquer le périple et identifier les bons et les méchants, n'est pas même systématiquement employée : dans le baptême, la conversion anthroponymique apparaît moins essentielle que la dation du nom et celui-ci n'est pas obligatoirement clairement chrétien, pourvu qu'il soit donné au baptême: l'enfant trouvé sous un frêne, peut bien être nommé Frêne pourvu que ce soit sur les fonts baptismaux ${ }^{32}$.

17 Il n'empêche, les choix finissent par se concentrer toujours plus fortement sur des noms qui figurent bien au panthéon de l'Église et qui servent d'exemples aux directives ecclésiastiques.

\section{Pierre et Jean : l'engouement et le modèle}

18 Dans le nord de la péninsule ibérique, Pedro et, dans une moindre mesure Juan pour les hommes, Maria pour les femmes, s'imposent dans presque toutes les régions à partir du milieu du XIe siècle ${ }^{33}$; dans la péninsule italienne, « la palme absolue revient partout et toujours à Jean $»^{34}$; dans le domaine français, c'est « d'abord Pierre qui monte en flèche après 1050 , acquiert la prépondérance au XII ${ }^{e}$ pour atteindre son apogée vers la fin du premier tiers du XIVe siècle. La percée de Jean se fait à partir des années 1250 : elle est fulgurante et installe définitivement le nom en tête des choix jusqu'au XVIII siècle ${ }^{35}$.

Jean et Pierre figurent à titre d'exemple dans les formulaires de baptême dont les synodes du XIII siècle multiplient les énoncés ${ }^{36}$. Il peut s'agir d'un simple effet en retour d'usages déjà en vogue mais aussi d'une de ces incitations que l'Église n'a plus alors cessé d'adresser aux fidèles. Les actes conciliaires et les rituels se préoccupent en effet toujours plus et plus précisément du nom de baptême. Il ne s'agit toujours que de conseils, comme au temps du Concile de Trente et de la défense du catholicisme contre les déviances quand les rituels désapprouvaient l'emploi des noms de l'Ancien Testament qui pouvaient faire confondre les Catholiques avec les Protestants et les $\mathrm{Juifs}^{37}$. Mais, via la prédication et le travail sacerdotal au quotidien, ils devaient bien agir sur la pratique anthroponymique et l'orienter.

20 Toujours est-il que les résultats sont là, frappants : avec Jean et Pierre, mais aussi Jacques, François, Dominique, Marie et quelques autres, tels Guillaume et Henri ici, Bertrand ou Raymond là, rescapés du stock germanique et désormais très chrétiens, le panthéon catholique est prépondérant dès le XIII ${ }^{e}$ siècle pour culminer au XVIII ${ }^{e}$ siècle et, preuve supplémentaire de la victoire éclatante du centralisme clérical, le mouvement délaisse largement les saints locaux, à quelques exceptions notables tel Claude en Jura ou Léonard en Limousin ${ }^{38}$.

\section{Le surnom libérateur}

21 La concentration des choix créait une homonymie forte qui, si elle satisfaisait les tenants de l'unicité du peuple de Dieu, gênait au civil la vie collective. Certes la châne de cause à effet n'est pas évidente à définir précisément, tant les situations sont foisonnantes d'une région à l'autre et délicates à explorer à partir d'une documentation 
longtemps lacunaire, mais il est bien difficile de ne pas voir une forte corrélation entre cette concentration des choix et la diffusion du surnom intervenant à peu près au même moment, après l'an mille chez les aristocrates, à partir du XII ${ }^{e}$ siècle chez les roturiers ${ }^{39}$. Perdant une partie de son rôle de classificateur social car libéré de cette fonction par le surnom, le nom de baptême pouvait alors se consacrer à la charge religieuse.

\section{Hypocoristiques et prénominations multiples : le renforcement des noms dominants} mort $»^{40}$. politique $^{41}$. additionner.

Partout les déformations hypocoristiques des noms et la création des noms doubles ont regonflé quelque peu les possibilités de choix mais sans augmenter la part de l'invention, en confortant au contraire le monopole des noms dominants.

Ainsi dans le Lyonnais du XIV ${ }^{e}$ siècle, le stock des prénoms est limité, le taux d'homonymie élevé : sur 4064 individus de sexe masculin, 17 noms suffisent à en nommer $82 \%$. Mais l'engorgement est bien atténué par le succès des formes dérivées qui parviennent à égaler, voire à dépasser en nombre les formes originelles. "Plutôt que d'introduire un nouveau nom, on déforme, on triture les noms déjà familiers. $\mathrm{Ce}$ foisonnement des noms raccourcis ou allongés est d'autant plus remarquable que ce ne sont pas des appellations provisoires, des diminutifs que l'on abandonne avec l'enfance: ce sont de véritables noms, conservés sous la même forme jusqu'à la

Quant à la pratique de la prénomination multiple et des prénoms doubles, elle n'est, au Moyen-Âge, vraiment attestée que sous la première forme et seulement en Toscane : $60 \%$ environ des quelques neuf cents enfants de familles bourgeoises florentines étudiées à travers la documentation familiale portent un deuxième prénom dans la période 1360-1530. Le premier, usuel et parental, reste traditionnellement assez étranger à la tradition chrétienne; le second, d'un usage limité semble-t-il, se réfère généralement au calendrier liturgique et une fois sur deux, c'est celui du saint dont la fête tombe le jour du baptême, une fois sur quatre il rappelle une dévotion familiale. Au $\mathrm{XV}^{\mathrm{e}}$ siècle, le système est envahi par un troisième prénom, celui de Romolo/Romola, un saint de la cité de Fiesole rattachée au début du siècle à l'évêché de Florence qui, en plaçant chaque citoyen sous son vocable, semble porter un véritable programme

À dire vrai, la prénomination multiple est aussi présente en France, dès le XIII ${ }^{\mathrm{e}}$ siècle, mais indirectement. Les canons synodaux stipulent en effet qu'« on ne doit pas admettre plus de trois personnes pour élever l'enfant au-dessus des fonts baptismaux car aller au-delà est une inspiration diabolique $»^{42}$. Il s'agit certes d'une interdiction mais molle on en conviendra, face à un usage qui paraît largement répandu : aux XIV ${ }^{e}$ et $\mathrm{XV}^{\mathrm{e}}$ siècles au moins, le milieu aristocratique la transgresse comme le montrent les chroniques; au XVIe siècle au plus tard d'après les registres paroissiaux, les roturiers n'en font guère de cas. Mais les effets anthroponymiques paraissent nuls car il n'est officiellement inscrit qu'un seul nom. Il reste que l'usage, ici caché, pouvait les

Inexistantes ou masquées, les pratiques des prénoms secondaires et composés ne sont donc pas attestées en France avant la fin du XVI ${ }^{e}$ siècle dans le Midi et au XVII ${ }^{e}$ siècle 
dans le Nord, la seconde forme plus tardivement encore que la première. Mais l'engouement est alors fulgurant et vient renforcer la position dominante des noms les plus usités : partout, Jean et Marie, très fortement présents comme prénoms uniques, voient leur importance augmenter encore comme "prénoms bases» des formes composées ${ }^{43}$.

\section{Le parrainage : entre clientèle terrestre et patronage céleste}

La documentation reste longtemps silencieuse et si la pratique est attestée pour l'époque carolingienne ${ }^{44}$, on ignore la manière dont elle s'intègre à ce moment aux usages familiaux. C'est plus clair à partir du XIII ${ }^{\mathrm{e}}$ siècle et tout montre alors que la parenté spirituelle sert la recherche de toutes les solidarités, aussi bien celles des clientèles terrestres que des patronages célestes. C'est dans le partage du sang, des intérêts mais aussi du nom que les solidarités se marquent et se soudent, ainsi que le revendique dans le roman en prose qui lui est consacré vers 1450 « le comte de Flandre qui se trouvait à la cour du roi Philippe $[. .$.$] : mon très cher seigneur, vous êtes mon$ parrain et je porte votre nom. Pour cette raison, par votre grâce, accordez-moi un don : celui d'aller secourir le marquis de Milan, de chasser les Sarrasins et de venger le SaintSiège apostolique $»^{45}$.

28 En fonction du rôle assigné à la parenté spirituelle dans le choix du nom, les mécanismes de la transmission esquissent une opposition entre les pays du Nord et ceux de la Méditerranée, dont on est encore loin, il est vrai, de pouvoir reconnaitre et de comprendre les rythmes et les contours, mais toutes les pratiques, à leur manière, permettent de préserver les intérêts anthroponymiques de la famille.

Un modèle plutôt exogamique semble régner surtout sur les zones méditerranéennes : parrains et marraines y sont choisis hors du cercle familial et ne donnent pas leur nom. Le cercle des amis, des alliés s'en trouve grandi, le réseau des alliances est étoffé sans que la cohérence du stock anthroponymique soit perturbée. Dans la Florence des XIV ${ }^{e_{-}}$ $\mathrm{XVI}^{\mathrm{e}}$ siècles, parenté naturelle et parenté spirituelle se juxtaposent et n'empiètent presque jamais l'une sur l'autre. Un pourcentage infime $(1,5 \%)$ des parrains et marraines appartient aux consanguins ou aux proches alliés des parents du baptisé, au moins dans les couches sociales dont on a conservé les « livres de famille ». L'immense majorité des parents spirituels d'enfants nés dans ces familles de la bourgeoisie, grande ou petite, de la ville, se recrute parmi les voisins, les collègues, les relations politiques ou d'affaires du père de l'enfant. Pour ce qui est du nom de leur filleul, les parrains n'ont pas d'autre rôle que de transmettre devant Dieu et devant le prêtre, au cours de la cérémonie du baptême à laquelle n'assistent pas les parents de l'enfant, celui ou ceux que ces derniers ont choisi(s) ${ }^{46}$.

30 Au Nord, dans le domaine français en tout cas, le modèle est plutôt endogame : parrains et marraines sont choisis dans le cercle familial et donnent leur nom. Sont ainsi resserrés les liens intra-familiaux, lignagers pour les aînés, latéraux pour les suivants, respectés et reconduits les stocks familiaux de nom.

31 Ici et là, ponctuellement, une troisième voie se dessine, comme en Gévaudan au $\mathrm{XV}^{\mathrm{e}}$ siècle. Les parrains et marraines sont ici choisis généralement hors de la parenté 
proche, donnent toutefois systématiquement ou presque leur nom: ils sont sélectionnés, par le père, entre autre pour le nom qu'ils portent, au moins pour l'aîné ${ }^{47}$.

Ainsi, la pratique qui veut que le parrain soit désigné par le père en fonction du nom qu'il porte, à l'intérieur ou à l'extérieur de la parenté naturelle, pour à la fois agrandir le cercle des protecteurs et préserver la cohérence du stock familial des noms, est en marche dès le XIII siècle au moins et s'impose pour devenir à la fin du Moyen Âge et aux Temps modernes, dans la littérature comme dans la réalité, une norme rarement transgressée ; au point que les programmes dévotionnels particuliers paraissent parfois réclamer une certaine recherche voire affectation et que les manquements sont soulignés. En 1377, pour répondre à «la dévocion que la royne avoit à Madame Sainte Katherine, [...] fut le parrain le prieur de Sainte-Katherine du Val des Escholiers de Paris et marraine une damoiselle qui aidait à dire les heures à la dite royne, appellée damoiselle Katherine de Villiers ", fille il est vrai de Pierre de Villiers, grand maître de l'hôtel du roi ${ }^{48}$. Et quand, en 1433, un fils de la duchesse de Bourgogne « fut sur lesdits fonts nommé Josse ", le chroniqueur Enguerrand de Monstrelet éprouve le besoin de dire que « jà-soit-ce que nul desdits parrains et marraines eussent ainsi nom, mais ainsi l'avoient ordonné lesdits duc et duchesse $»^{49}$.

La dénomination semble bien être le résultat d'un équilibre empiriquement façonné par la pratique entre les exigences familiales et sociales, profanes et ecclésiales. À un ou deux éléments, elle sert à identifier les individus, à les classer parmi la société, à les relier aux morts et à la divinité : car elle doit aussi bien protéger les individus face au monde que les rassurer devant l'éternité.

\section{NOTES}

1. B. This, "Nomination", Le nom et la nomination. Sources, sens et pouvoirs, ss. la dir. de Noël Clerget, Paris, 1990, p. 303.

2. O. Journet, «La personne au risque du nom », Le nom et la nomination..., op. cit., p. 189.

3. L. Perouas et alii, Léonard, Marie, Jean et les autres. Les prénoms en Limousin depuis un millénaire, éd. du CNRS, Paris, 1984, p. 86.

4. J. Corblet, «Des noms de baptême et des prénoms des chrétiens. Etude philologique et liturgique ", Revue de l'Art chrétien, t. V, 1876, p. 10.

5. V. Feroldi, « Dieu connaît chacun par son nom », Le nom et la nomination..., op. cit., p. 271.

6. Jean Wauquelin, La belle Hélène de Constantinople, roman en prose du milieu du $\mathrm{XV}^{\mathrm{e}}$ siècle, présenté par M.-Cl. de Crécy, Splendeurs de la cour de Bourgogne, récits et chroniques, Paris, 1995, p. 181.

7. «L'enfant dans le bas ", Les esprits et les morts, croyances médiévales, Cl. Lecouteux et Ph. Marcq éd., Honoré Champion/Essais, Paris, 1990, p. 101.

8. $\mathrm{P}$. Beck, «Discours littéraires sur l'anthroponymie (VI ${ }^{\mathrm{e}}-\mathrm{XVI}{ }^{\mathrm{e}}$ siècles) », Genèse médiévale de l'anthroponymie moderne, tome IV, Discours anthroponymiques ( $\mathrm{VI}^{e}-\mathrm{XVI} I^{e}$ siècles), P. Beck dir., Université de Tours, 1997, pp. 121-161.

9. Évangile selon Saint Luc, Chap. 1, verset 59-63 : circoncision de Jean le Baptiste. 
10. R. Le Jan, « Nommer/identifier ou la puissance du nom dans la société du haut Moyen-Âge ", Des noms et des hommes, Sources Travaux historiques, $\mathrm{n}^{\circ}$ 45-46, pp. 47-56.

11. La chanson des Nibelungen, traduction intégrale avec introduction et notes par M. Colleville et E. Tonnelat, Aubier-Montaigne, Paris, 1944, respectivement 140-192 et 20-21.

12. Génèse médiévale de l'anthroponymie moderne I, M. Bourin dir., Université de Tours, 1989 ; idem II-1 et II-2, M. Bourin et P. Chareille dir., Université de Tours, 1992; "Genèse médiévale de l'Anthroponymie moderne : l'espace italien », J.-M. Martin et F. Menant dir., Mélanges de l'Ecole Française de Rome, Moyen Âge,vol. 1, t. 106-2-1994, pp. 313-736; idem,vol. 2, t. 107-2-1995, pp. 331-633 ; idem,vol. 3, t. 110-1-1998, pp. 79-270 ; Antroponomia y sociedad. Sistemas de identification hispano-cristianos en los siglos IX a XIII, P. M. Sopena dir., Universidad de Santiago de Compostella y Valladolid, 1995 ; L'anthroponymie, document de l'histoire sociale des mondes médiévaux méditerranéens. Actes $d u$ colloque organisé par l'École française de Rome avec le concours du GDR $955 d u$ CNRS (Rome, 6-8 octobre 1994), M. Bourin, J.-M. Martin et F. Menant dir., Rome, 1996.

13. Genèse médiévale de l'anthroponymie moderne III, enquêtes généalogiques et données prosopographiques, M. Bourin et P. Chareille dir., Université de Tours, 1995.

14. Chrétien de Troyes et ses continuateurs (Manuscrit de Mons), Perceval ou le roman du Graal,traductions et notes de J.-P. Fouchais et A. Ortais, Paris, 1974, p. 44.

15. Jean d'Arras, Le roman de Mélusine ou l'histoire des Lusignan, mis en français moderne par M. Perret, Paris, 1979, p. 178.

16. L. Perouas et alii, Léonard, Marie, Jean et les autres..., op. cit., p. 35.

17. Ch. Klapisch-Zuber, «Le nom refait. La transmission des prénoms à Florence (XIV ${ }^{\mathrm{e}}-\mathrm{XVI}^{\mathrm{e}}$ siècles) ", L'Homme, oct.-déc. 1980, XX (4), pp. 77-104.

18. J.-L. Biget, «L'évolution des noms de baptême en Languedoc au Moyen Âge (IX $\left.{ }^{\mathrm{e}}-\mathrm{XIV}^{\mathrm{e}} \mathrm{s}.\right)$ ", Liturgie et musique, Cahiers de Fangeaux $n^{\circ}$ 17, Toulouse, 1982, p. 299.

19. A. Fine, "L'héritage du nom de baptême ", Annales. Économie, Société, Civilisation, 4, 1987, p. 862.

20. La chanson des Nibelungen, op. cit., 716 et 718.

21. Le livre de Baudoin, comte de Flandre, op. cit., pp.89-90.

22. Jean Wauquelin, La belle Hélène de Constantinople, roman en prose, 1448, présenté par M.-Cl. de Crécy, Splendeurs de la cour de Bourgogne, récits et chroniques, Paris, 1995, p. 200.

23. Idem, p. 118.

24. Le livre de Baudoin, op. cit, pp. 89-90.

25. A. Burguière, « Un nom pour soi. Le choix du nom de baptême en France sous l'Ancien Régime (XVI ${ }^{\mathrm{e}}$-XVIII ${ }^{\mathrm{e}}$ siècles) », L'Homme, oct.-déc. 1980, XX (4), pp. 26-27.

26. Cf. Génèse médiévale de l'anthroponymie moderne... I, II, III, op. cit.

27. A. Lefèvre-Teillard, Le nom, droit et histoire, Paris, PUF, 1990.

28. J. Corblet, op. cit., p.15.

29. Cité dans J. Dupaquier, «Le prénom, approche historique », Le nom et la nomination..., op. cit., pp. 209-222.

30. J.L. Biget, op. cit., p. 308.

31. J. Corblet, op. cit., p. 7.

32. P. Beck, « Discours littéraires sur l'anthroponymie... », op. cit, p. 125.

33. Antroponomia y sociedad..., op. cit.,pp. 396-397.

34. « Genèse médiévale de l'Anthroponymie moderne : l'espace italien 1 », op. cit., p. 734.

35. Ainsi que Perrette et Jeanne pour les femmes : J.-L. Biget, op. cit., p. 329.

36. Les statuts synodaux français $d u X^{X} I I^{e}$ siècle, t. 1, Les statuts de Paris et le synodal de l'ouest, publiés et traduits par Odette Pontal, Paris, Bibliothèque Nationale, 1971.

37. J. Corblet, op. cit., p. 275.

38. L. Perouas et alii, Léonard, Marie, Jean et les autres... op. cit., p. 56 sq.

39. Genèse médiévale de l'anthroponymie moderne... I, II, op. cit. 
40. F. Maillard, « Noms de personne du Lyonnais en 1307 », Actes du $89^{\mathrm{e}}$ congrès national des Sociétés Savantes, Lyon, 1964, Bulletin Philologique et Histotrique,LX-1964, pp. 735-770.

41. Ch. Klapisch, « Le nom refait », op. cit., p. 81.

42. Les statuts synaudaux français $d u$ XIII ${ }^{e}$ siècle..., op. cit.

43. A. Burguière, op. cit. p. 29.

44. M. Rubelin, « Entrée dans la vie, entrée dans la chrétienté, entrée dans la société : autour du baptême à l'époque carolingienne ", Entrer dans la vie, passages et apprentissages au Moyen Âge,XII congrès de la SHMESP, Nancy, 1981, Annales de l'Est, 1982, n 1-2, pp. 31-51.

45. Le livre de Baudoin, comte de Flandre, roman en prose anonyme, présenté par D. Régnier-Bohler, Splendeurs de la cour de Bourgogne, récits et chroniques, Paris, 1995, p. 10.

46. Ch. Klapisch, «Parrains et filleuls. Une approche comparée de la France, de l'Angleterre et de l'Italie médiévales », Medieval Prosopography, 1986, vol. 6, pp. 51-77.

47. Ph. Maurice, "L'état civil des notaires du Gévaudan à la fin du Moyen-Âge: choix des parrains, choix des noms ", Genèse médiévale de l'anthroponymie moderne, tome IV, Discours anthroponymiques (VI'-XVI $I^{e}$ siècles), op. cit., pp. 179-209.

48. Les Grandes chroniques de France. Chronique des règnes de Jean II et de Charles V, 1350-1380,publiée par R. Delachenal, 2 vol., Paris, 1910-1916, vol. 2, p. 277.

49. Enguerrand de Monstrelet, Chroniques (1400-1444), éd. Douët d'Arecq, livre II, chap. CXXXII,Paris, 1857-1862.

\section{AUTEUR}

\section{PATRICE BECK}

Université de Paris-I Panthéon-Sorbonne 